\title{
Practice
}

Abbs, I.C. c98

Abo-Zenah, H. c108

Afzali, B. c98

Almirall, J. c17

Almond, M.K. c83

Altmann, P. c41

Antoljak, N. c103

Ayuso, S. c118

Barreto, G.P. c6

Beaman, M. c142

Bešlin, M.B. c103

Betriu, A. c17

Bex, S. c108

Boaz, M. c67

Bonomo, Y. c41

Bossola, M. c76

Brindo, M. c118

Brown, E.A. c3

Cairns, T. c154

Cánepa, C. c118

Carreño, A. c125

Cascino, A. c76

Chagnac, A. c67

Chang, S.-T. c90

Chen, C.-C. c90

Chen, C.-L. c90

Chi, C.-S. c137

Cooper, R. c41

Davenport, A. c33

Dhaygude, A. c154
Dhillon, S. c83

Dieguez, S. c118

Dohi, Y. c49

D'Souza, R.J. c142

El Nahas, A.M. c108

Endo, M. c147

Evenepoel, P. c1

Fu, L.-S. c137

Fujimi, S. c23

Fujita, T. c147

Fuke, Y. c147

Gafter, U. c67

Gallar, P c125

Goldsmith, D.J.A. c98

Graham, K. c41

Griffin, M. c108

Griffith, M. c154

Haase, M. c131

Haydar, A.A. c98

Heitzler, V.N. c103

Herman, M. c67

Hidaka, M. c147

Hirakata, E. c23

Hirakata, H. c23

Huh, W. c5

lida, M. c23

Ishikawa, I. c11

Johnson, T.S. c108

Kanai, H. c23

Kang, W.-H. c5
Kashiwagi, M. c23

Kim, B. c5

Kim, D.-J. c5

Kim, Y.-G. c5

Klinger, R. c103

Kojima, M. c49

Krausch, D. c131

Krizmanić-Dekanić, A. c103

Kubo, M. c23

Laviano, A. c76

Lee, K.-B. c5

Lee, Y.-H. c5

Liberatori, M. c76

Lin, F.-C. c90

Llibre, J. c17

Lopez, T. c17

Lozano, L. c125

Luciani, G. c76

Mazul-Sunko, B. c103

McFarlane, P.A. c35

McLean, A. c154

Melzer, C. c131

Mizumasa, T. c23

Molina, A. c125

Morgera, S. c131

Moussier, M.L. c76

Muñoz, M. c125

Muscaritoli, M. c76

Nash, M.M. c35

Neumayer, H.-H. c131
Neves, J. c61

Newstead, C.G. c31

Nicholls, A.J. c142

Oh, H.-Y. c5

Ohi, H. c147

Ohsawa, I. c147

Oliet, A. c125

Ori, Y. c67

Ortega, O. c125

Ortiz, M. c125

Osinde, E. c118

Palmer, A. c154

Panocchia, N. c76

Plata-Salaman, C.R. c73

Prasad, G.V.R. c35

Rajagopalan, B. c41

Rodríguez, I. c125

Rosseau, S. c131

Rossi Fanelli, F. c76

Ruiz, A. c17

Siranović, M. c103

Satchell, S.C. c142

Sato, K. c49

Sawaya, A.L. c6

Schmilovitz-Weiss, H. c67

Scholle, C. c131

Sesso, R. c61

Shien, C.-Y. c137

Širanović, M. c103

Skill, N.J. c108
Stompór, T.P. c123

Styles, P. c41

Tamano, M. c147

Tanaka, H. c23

Taniguchi, M. c49

Taube, D. c154

Taylor, D.J. c41

Tazza, L. c76

Ueda, R. c49

Valenza, V. c76

Vallve, M. c17

Vargas-Hein, O. c131

Vaux, E.C. c41

Vigil, A. c125

Voss, G. c131

Vrkić, N. c103

Weinstein, T. c67

Wild, G. c108

Worrall, S.P.R. c83

Wu, D. $c 90$

Yoon, S.-J. c5

Yoshimitsu, T. c23

Zaltzman, J.S. c35

Žarković, N. c103

Zevin, D. c67

Zuckermann-Becker, H. c131

\section{Subject Index Vol. 97, 2004}

Acquired renal cystic disease c11 Acute renal failure $\mathrm{c} 103$

ANCA-associated vasculitis c154 Anorexia c76

Anticoagulation c5

Antineutrophil cytoplasmic autoantibody $\mathrm{c} 142$

Atrial natriuretic peptide $\mathrm{c} 103$

Birth weight c61

Bland-Altman analysis c 98

Blood pressure c61

Brain atrophy c23

Bupropion c83

Calcineurin inhibitors, questionnaire c35

Calcium channel blocker c49

Carnitine c41

Cholesterol $\mathrm{c} 17$

Chronic hemodialysis, quality of life c90

- $\quad$ kidney failure c108

renal failure $\mathrm{c} 103$

Citrate anticoagulation c131

Complement c147

Computed tomography $\mathrm{c} 11$

Continuous cyclophosphamide c154

- renal replacement therapy $\mathrm{c} 131$

C-reactive protein $\mathrm{c} 125$

Creatinine clearance $\mathrm{c} 118$

Critically ill septic patient $\mathrm{c} 103$
Cyclosporine c 35

Cystatin C c103

Diabetic nephropathy c108

Dialysis c67

Dialysis-related hypotension c23

Dinamap c98

Echocardiography c90

Elderly patients c142

End-stage renal disease c76

Factor H c147

Fibrosis c108

Frontal lobe atrophy c23

Glomerular filtration rate $\mathrm{c} 118$

Haemodialysis c83

HBV c67

Hemodialysis c90

-, hypotension $\mathrm{c} 23$

Hemolytic-uremic syndrome c118

Hemophan $\mathrm{c5}$

Heparin $\mathrm{c} 5$

Histology c11

24-Hour ambulatory blood pressure monitoring c49

Hydroxybupropion c83

Hyperphosphataemia $\mathrm{c} 17$

Hypertension c61

Immunogenicity c67

Immunosuppressive therapy, side effects c 35

Inferior vena cava diameter $\mathrm{c} 90$
$\mathbf{K t} / \mathrm{V}_{\text {urea }}$ c 90

$\mathbf{L}$-Carnitine, treatment c41

Lacunar infarction c23

Leptin c76

Levamisole c137

Likert scale c35

Membranous nephropathy c147

Metabolic complications c13

Metabolites c83

Microscopic polyangiitis $\mathrm{c} 142$

Muscle bioenergetics c41

Myopathy c41

$\mathbf{N}$-terminal pro-B-type natriuretic peptide $\mathrm{c} 125$

Oliguria $\mathrm{c} 118$

Omron c98

Papillary renal cell carcinoma $\mathrm{c} 11$

Pharmacokinetics c83

Phosphate binders $\mathrm{cl}$

Phosphorus magnetic resonance spectroscopy $\mathrm{c} 41$

Pre-dialysis patients $\mathrm{c} 125$

proANP(1-98) c103

Prognosis c11

Protein load c118

Protein-energy malnutrition c61

Proteinuria c118

Proton efflux c41

Pulse cyclophosphamide c154

Questionnaire c11
Random-zero c98

Renal cell carcinoma c11

- failure c41

- functional reserve c118

transplant recipient experience, survey c35

- transplantation c35

vasculitis c142

Scarring c108

Screening c11

Sevelamer c17

SF-36 c90

Skeletal muscle c4

Steroid dependency c137

Steroid-sensitive nephrotic syndrome c137

Tacrolimus c35

Threohydrobupropion c83

Transglutaminase $\mathrm{c} 108$

Undernutrition c61

Vaccine c67

Volume control c125

Wegener's granulomatosis c142

\section{KARGER}

ㄷ) 2004 S. Karger AG, Basel

Fax +41613061234

E-Mail karger@karger.ch

www. karger.com

Accessible online at: www. karger.com/nec 